ИЗВЕСТИЯ АКАДЕМИИ НАУК ЭСТОНСКОИ ССР. ТОМ 25 ФИЗИКА * МАТЕМАТИКА. 1976, № 3

\title{
КОРРЕЛЯЦИОННАЯ ФУНКЦИЯ ТЕМПЕРАТУРЫ ТАЮЩЕГО ГРУНТА И ОБОБЩЕНИЕ МЕТОДА СТАТИСТИЧЕСКОЙ ЛИНЕАРИЗАЦИИ
}

В [1] при оценке теплового баланса тающей подстилающей поверхности основное внимание уделялось вычислению количества затрачиваемого на таяние тепла. В настоящей работе, исходя из той же самой модели тающего грунта и прежних условий случайно меняющегося освещения (переменная облачность), подробнее рассмотрены соответствующие случайные изменения температуры в почве.

\section{1. Температура тающего грунта}

Наложим на модель грунта следующие ограничительные условия:

1. Поглощение солнечной энергии происходит в тонком поверхностном слое грунта.

2. Система состоит практически из двух слоев - воздуха и неталого грунта.

Пусть поток прямой солнечной радиации $S(t)$ есть стационарная случайная функция от времени с постоянным средним значением $\bar{S}$, т. е. принятый режим освещения продолжается бесконечно долго. Тогда тепловой режим в почве можно тоже считать стационарным: вся почва теоретически до бесконечности приобретает одинаковую среднюю температуру $\bar{\Theta}$, которая не зависит ни от времени, ни от глубины.

Сравним модель тающего грунта со случаем, когда все заданные параметры почвы и условия осве-

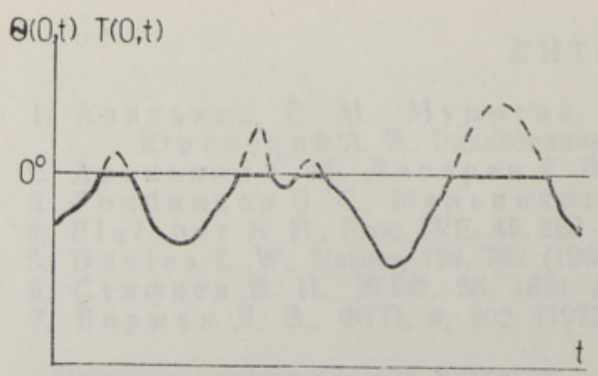

Рнс. 1. Реализации температуры земной поверхности для тающего и нетающего (-- -) грунтов. щения остаются в силе, но грунт не тает. Температуру поверхности нетающего грунта обозначим через $T(0, t)$ и предположим, 'что она является гауссовым процессом со средним значением $\bar{T}$ и дисперсией $\sigma_{T}^{2}$.

Далее, допустим, что возникающая при таянии вода полностью удаляется с поверхности. Тогда температура грунта будет отличаться от $T(0, t)$ лишь тем, что у нее на высоте $0^{\circ} \mathrm{C}$ пропадут максимумы (рис. 1). 
Если температуру измерять по шкале Цельсия, то случайная функция $\Theta(0, t)$ примет вид

$$
\Theta(0, t)=T(0, t) U[-T(0, t)],
$$

где

$$
U[x]= \begin{cases}0, & x<0 \\ 1, & x>0\end{cases}
$$

Поскольку функция распределения $p(T)$ считалась нормальной, выразить $\bar{\Theta}$ и $\sigma_{\Theta}^{2}$ через $\bar{T}$ и $\sigma_{T}^{2}$ оказывается несложно:

$$
\begin{aligned}
& \bar{\Theta}=-\frac{\sigma_{T}}{\sqrt{2 \pi}} e^{-\frac{\bar{T}^{2}}{2 \sigma_{T}^{2}}}+\bar{T} \Phi\left(\frac{-\bar{T}}{\sigma_{T}}\right) \\
& \sigma_{\Theta}^{2}=-\bar{T} \frac{\sigma_{T}}{\sqrt{2 \pi}} e^{-\frac{\bar{T}^{2}}{2 \sigma_{T}^{2}}}+\left(\sigma_{T}^{2}+\bar{T}^{2}\right) \Phi\left(\frac{-\bar{T}}{\sigma_{T}}\right)-\bar{\Theta}^{2},
\end{aligned}
$$

где

$$
\Phi(x)=\frac{1}{\sqrt{2 \pi}} \int_{-\infty}^{x} \mathrm{e}^{-\frac{\eta^{2}}{2}} \mathrm{~d} \eta .
$$

Согласно разработанной в $\left[{ }^{2}\right]$ методике, статистические характеристики поверхностной температуры нетающего грунта $\bar{T}$ и $\sigma_{T}^{2}$, а также корреляционную функцию $R_{T}(\tau)$ можно связать с соответствующими статистическими параметрами падающего на поверхность почвы потока солнечной радиации. Для этого необходимо знать альбедо подстилающей поверхности, условия испарения воды с поверхности почвы и коэффициенты температуропроводности и теплоемкости грунта и воздуха.

Исследуем полученные выражения.

Если $\bar{T} \rightarrow-\infty$, то $\bar{\Theta}=\bar{T}$ и $\boldsymbol{\sigma}_{\boldsymbol{\theta}}^{2}=\sigma_{T}^{2}$. Средняя температура грунта столь низка, что максимумы флуктуаций не достигают точки таяния. Максимумы функции $T(0, t)$ не пропадают.

Если $\bar{T}=0^{\circ}$, то $\bar{\Theta}=-\sigma_{T} / \sqrt{2} \pi$ и $\sigma_{\Theta}^{2}=\sigma_{T}^{2}(\pi-1) / 2 \pi$. Это значит, что когда поверхностная температура нетающего грунта в среднем равна нулю, средняя температура тающего грунта ниже нуля. Понижение средней температуры зависит от дисперсии $\sigma_{T}^{2}$, которая, в свою очередь, зависит от характера изменчивости падающей на поверхность грунта радиации. По сравнению с дисперсией нетающего грунта дисперсия температуры при таянии уменьшается.

Если $\vec{T} \rightarrow \infty$, то $\bar{\Theta}=0^{\circ}$ и $\sigma_{\Theta}^{2}=0$. Температура поверхности тающего грунта не поднимается выше нуля.

\section{2. Приближенные выражения для корреляционной функции; обобщенный метод статистической линеаризации}

Приступим к рассмотрению корреляционной функции $R_{\Theta}(\tau)$. Основная формула для ее вычисления следующая:

$$
R_{\Theta}(\tau)=\int_{-\infty}^{\infty} \int_{-\infty}^{\infty} \Theta(t) \Theta(t+\tau) p\left(T_{1}, T_{2}\right) \mathrm{d} T_{1} \mathrm{~d} T_{2}-\bar{\Theta}^{2},
$$


где $T_{1}=T(0, t), T_{2}=T(0, t+\tau)$, а $p\left(T_{1}, T_{2}\right)$ - двумерное гауссовое распределение.

Известно, что распределение $p\left(T_{1}, T_{2}\right)$ разложимо в ряд по степеням нормированной корреляционной функции температуры $T\left[{ }^{3,4}\right]$. Тогда корреляционную функцию температуры $\Theta$ можно выразить тоже рядом

$$
R_{\Theta}(\tau)=\sum_{i=1}^{\infty} \sigma_{T}^{2}\left[\Phi^{(i-1)}\left(\frac{-\bar{T}}{\sigma_{T}}\right)\right]^{2} \frac{r_{T}^{i}(\tau)}{i !},
$$

где $r_{T}(\tau)$ - нормированная корреляционная функция температуры $T$.

Ограничение лишь первыми членами ряда (3) дает для $R_{\Theta}(\tau)$ следующие приближения:

$$
\begin{aligned}
& R_{1 \Theta}(\tau)=\left[\Phi\left(\frac{-\bar{T}}{\sigma_{T}}\right)\right]^{2} R_{T}(\tau) \\
& R_{2 \Theta}(\tau)=\left[\Phi\left(\frac{-\bar{T}}{\sigma_{T}}\right)\right]^{2} R_{T}(\tau)+\frac{\sigma_{T}^{2}}{4 \pi} e^{-\frac{\overline{T^{2}}}{\sigma_{T}^{2}} r_{T}^{2}(\tau)} \\
& R_{n \Theta}(\tau)=\sum_{i=1}^{n} \sigma_{T}^{2}\left[\Phi^{(i-1)}\left(\frac{-\bar{T}}{\sigma_{T}}\right)\right]^{2} \frac{r_{T}^{i}(\tau)}{i !} .
\end{aligned}
$$

Чтобы понять сущность такого довольно формального метода, вычислим $R_{\Theta}(\tau)$ еще другим способом.

Перепишем выражение (1) в виде $\Theta=g(T)$ и рассмотрим его как описание некоторой системы, входной функцией которой является $T$, а выходной $-\Theta$. Передаточная функция $g$ недифференцируема и имеет вид, изображенный на рис. 2.

Заменим нелинейную передаточную функцию $g$ многочленом $n$ порядка

$$
g_{n}(T)=\sum_{i=0}^{n} a_{i}[T(t)-\bar{T}]^{i}
$$

Так как $g(T)$ недифференцируема, то коэффициенты $a_{i}$ не могут быть коэффициентами Тейлора. Для их определения предъявим требование, чтобы квадратичное отклонение между $g$ и $g_{n}$ было минимальным:

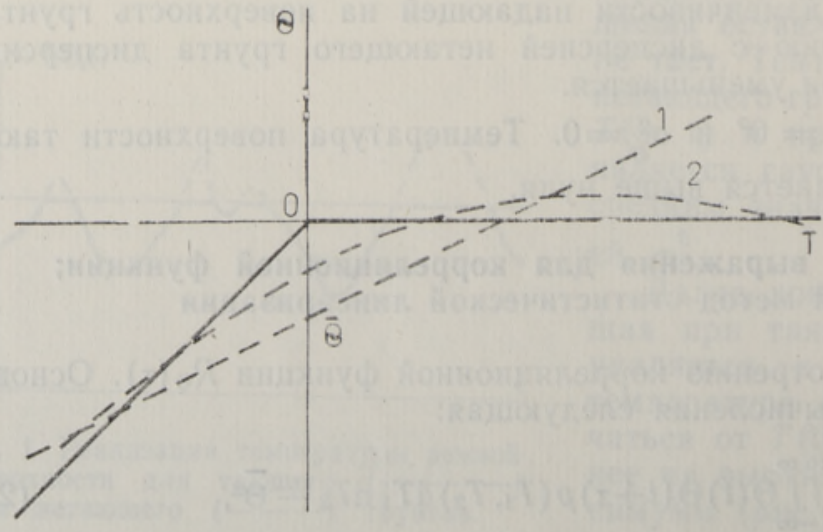

Рис. 2. Точная $(-)$ и
приближенные передаточные функция: $1-n=1,2-n=2$, $\bar{T}=0^{\circ}, \sigma_{T}=5^{\circ}$. 


$$
\left.\overline{\left[g(T)-g_{n}(T)\right.}\right]^{2}=\min .
$$
ний:

Это требование дает для определения $a_{i}$ систему из $n+1$ уравне-

$$
\sum_{j=0}^{n} a_{j} \mu_{(i+j), T}-\overline{g(T)[T-\bar{T}]^{i}}=0, \quad i=0,1,2, \ldots, n
$$

где $\mu_{(k), T}$ - центральный одномерный момент функции $T$.

Если известны коэффициенты $a_{i}$, то корреляционная функция $R_{\Theta}$ вычисляется по формуле

$$
R_{\Theta}(\tau)=\sum_{i=0}^{n} \sum_{j=0}^{n} a_{i} a_{j} \mu_{i j, T}-\bar{\Theta}^{2},
$$

где $\mu_{i j, T}$ - центральный двумерный момент функции $T$.

Так как процесс $T$ был принят гауссовым, то $\mu_{i j, T}$ можно выразить через степени корреляционной функции $R_{T}(\tau)$ и при $n \rightarrow \infty$ ряд (5) совпадет с рядом (3). Это значит, что учитывая в ряде (3) лишь первые члены, мы по существу заменяем передаточную функцию $g$ подходящим полиномом соответствующего порядка: при использовании приближения $R_{1 \Theta}$ ломаную кривую на рис. 2 заменяем некоторой прямой, при использовании $R_{2 \Theta}$ - параболой и т. д.

Надо сказать, что замена нелинейной передаточной функции некоторой статистически ей равноценной линейной известна в теории автоматического регулирования как метод статистической линеаризации Казакова $\left[{ }^{5,6}\right]$. То, что метод статистической линеаризации в случае гауссового входа представляет собой прекращение ряда (3) при $i=1$, отмечалось также в $\left[{ }^{7}\right]$. Развитый в настоящей работе метод можно назвать обобщением метода статистической линеаризации. Он может быть использован и в случае негауссовой входной функции. К достоинствам метода следует отнести то, что он позволяет вычисление интеграла (2) заменить вычислением двумерных моментов входной функции $T$ :

$$
\mu_{i j, T}=\int_{-\infty}^{\infty} \int_{-\infty}^{\infty}\left(T_{1}-\bar{T}\right)^{i}\left(T_{2}-\bar{T}\right)^{j} p\left(T_{1}, T_{2}\right) \mathrm{d} T_{1} \mathrm{~d} T_{2}
$$

Интегрирование $\mu_{i j, T}$ обычно значительно легче, чем интегрирование $R_{\Theta}(\tau)$ по основной формуле $(2)$, так как в (6) под интегралом стоит произведение двух степенных функций, а в (2) - произведение более сложных нелинейных функций $\Theta(t) \Theta(t+\tau)$.

\section{3. Свойства корреляционной функции}

Вернемся к изучению корреляционной функции $R_{\Theta}(\tau)$ и рассмотрим приближенные выражения (4). Так как в точной формуле (3) все члены ряда положительны, то приближения $R_{n \Theta}$ дают для корреляционной функции температуры $\Theta$ заниженные значения. Графики первых трех приближений показаны на рис. 3. Вычисления для графиков проведены при двух значениях $\bar{T}: \bar{T}=-\sigma_{T}=-5^{\circ}$ и $\bar{T}=\sigma_{T}=5^{\circ}$. Корреляционная функция температуры $T$ принималась экспоненциальной

$$
r_{T}(\tau) \approx \mathrm{e}^{-\alpha|\tau|}
$$

Это вполне допустимо по следующим соображениям. Установлено, что такой экспоненциальный вид имеет нормированная корреляционная функция падающей в условиях переменной облачности на земную поверхность солнечной радиации, причем $\alpha$ принимает значения 0,27 $-0,4$ мин $^{-1}\left[{ }^{8}\right]$. Если предположить, что теплопроводность почвы и 


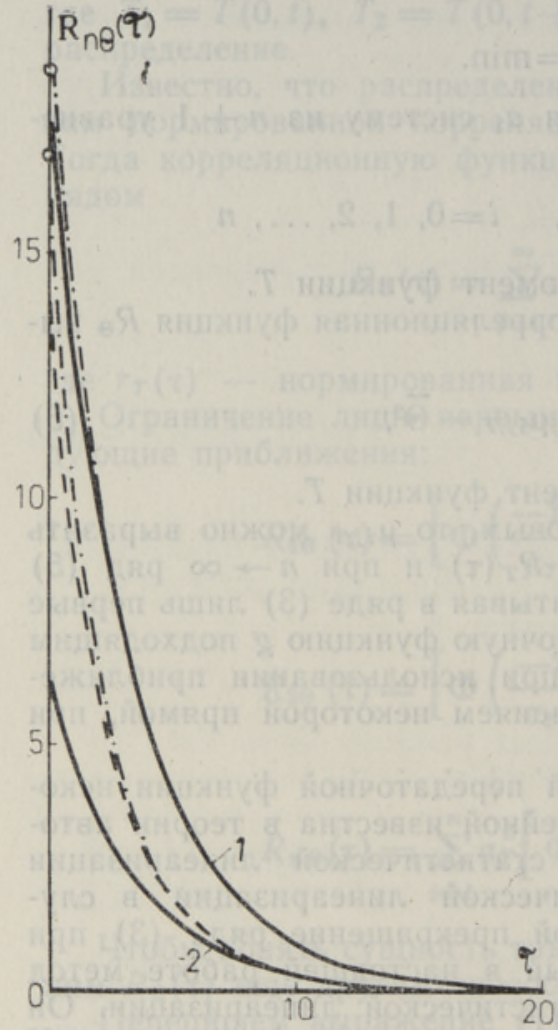

Рис. 3. Приближенные корреляционные функции поверхностной температуры тающего грунта: $1-\bar{T}=$ $=-5^{\circ}, 2-\bar{T}=5^{\circ}$ (масштаб увеличен в 10 раз); $\sigma_{T}=5^{\circ}$, $n=1$, - - $n=2,-\ldots-n=3$. Точные значения дисперсии обозначены кружками. приземного слоя воздуха пренебрежимо мала, то нормированные корреляционные функции радиации и поверхностной температуры будут приближенно одинаковы $\left[{ }^{2}\right]$, а выражение (7) в таких условиях оправдано. В настоящих расчетах для $\alpha$ выбрано значение 0,3 .

Формулы (4) и рис. 3 показывают, что приближение $R_{1 \Theta}$ довольно грубое. Вычисленная по $R_{1 \Theta}$ дисперсия $R_{1 \Theta}(0)$ заметно отличается от действительного ее значения, отмеченного на рис. 3 кружком. Приближения $R_{2 \Theta}$ и $R_{3 \Theta}$, видимо, аппроксимируют корреляционную функцию $R_{\Theta}(\tau)$ лучше. Они мало различаются между собой, и даваемые ими приближенные дисперсии незначительно отличаются от действительных.

Если нам известен температурный режим поверхности тающего грунта, то используя выработанную в [ $\left.{ }^{2}\right]$ методику, можно получить сведения и о флуктуациях температуры в глубине грунта. Для этого надо перейти от корреляционной функции $R_{\Theta}(\tau)$ к спектральной плотности $S_{\Theta}(\omega, 0)$ :

$$
S_{\Theta}(\omega, 0)=\frac{1}{2 \pi} \int_{-\infty}^{\infty} R_{\Theta}(\tau) \mathrm{e}^{-j \omega \tau} \mathrm{d} \tau,
$$

где $\quad \omega-$ частота, a j - мнимая

единица.

Тогда спектральная плотность для температуры внутри грунта выразится очень несложным образом:

$$
S_{\Theta}(\omega, z)=\mathrm{e}^{-\sqrt{\frac{2|\omega|}{k}} z} S_{\Theta}(\omega, 0),
$$

где $z$ - глубина в почве и $k$ - коэффициент температуропроводности грунта,

Применяя к $S_{\Theta}(\omega, z)$ преобразование Фурье, можно при желании получить отсюда и корреляционную функцию для температуры в глубине тающего грунта. 


\section{Л И Т Е Р А Т Р А}

1. Кээваллик С. Х., В сб.: Облачность и радиация, Тарту, 1975, с. 235.

2. Кээваллик С. Х., Муллама а Ю.-А. Р., Облачность и радиация, Тарту, 1975 , c. 212.

3. Тихонов В. И., Выбросы случайных процессов, М., 1970.

4. Тихонов В. И., Статистическая радиотехника, $M, 1966$.

5. П уг а е в В. С., Теория случайных функций, М., 1962.

6. К а з а к о в И. Е., Автоматика и телемеханика, № 5, 385 (1956).

7. П я тниц ки й Г. И., Автоматика и телемеханика, № 4, 474 (1960).

8. Стохастическая структура полей облачности и радиации, Тарту, 1972.
Институт термофизики и электрофизики Академии наук Эстонской ССР
Поступила в редакцию 25/IX 1975

\section{Sirje KEEVALLIK}

\section{SULAVA PINNASE TEMPERATUUR: KORRELATSIOONIFUNKTSIOON JA STATISTILISE LINEARISEERIMISE MEETODI OLDISTAMINE}

Vaadeldakse külmunud pinnase mudelit juhul, kui päikesekiirguse muutumist saab kirjeldada statsionaarse juhusliku funktsiooniga. Sulava aluspinna temperatuuri statistilised parameetrid (keskväärtus, dispersioon, korrelatsioonifunktsioon) seostatakse samasuguse, kuid mittesulava aluspinna vastavate parameetritega. Viimased on omakorda seoses juhusliku kiirgusrežiimi iseloomuga. Korrelatsioonifunktsioon arvutatakse kahel viisil: kasutades kahedimensioonilise normaaljaotuse rittaarendust ja üldistades Kazakovi statistilise lineariseerimise meetodit. Selgub, et nimetatud meetodid on ekviva. lentsed.

\section{Sirje KEEVALLIK ,}

\section{THE CORRELATION FUNCTION OF THE TEMPERATURE OF THAWING SOIL AND A GENERALIZATION OF THE METHOD OF STATISTICAL LINEARIZATION}

A model of frozen soil has been studied when the radiation regime can be described by a stationary random function. The statistical characteristics of the temperature of thawing soil (mean value, dispersion and correlation function) have been expressed by similar characteristics of the temperature of an analogous non-thawing model, which in their turn are connected with the random radiation regime. To calculate the correlation function, two methods have been used: development in a series of the two-dimensional normal distribution function and generalization of the Kazakov method of statistical linearization. It has been shown that both methods are equivalent. 